\title{
TRENDS IN REHABILITATION ROBOTS IN THE UNITED STATES
}

\author{
Kazuhiko Kawamura*
}

\begin{abstract}
This article presents an overview of rehabilitation robots in the United States. First, past history of robot usage in rehabilitation is reviewed. Then key $R \& D$ issues in planning, dexterous manipulation, sensing and reflex control are described. Finally future trends in user interface and learning are presented. One prototype rehabilitation robotic system under development at Vanderbilt University is used to illustrate key development issues.
\end{abstract}

\section{INTRODUCTION}

Rehabilitation engineering in the United States began after World War II through the Prosthetic and Sensory Aids Program by the Veterans Administration $^{1)}$. In this program engineers worked together with prosthetists and physicians to significantly advance the state of the art in artificial limbs. Since then many new areas of research have developed, one of which is robot usage.

The idea of employing robots in rehabilitation began in late 1970s. For example, Leifer et al. at Stanford University and the Veterans Administration Medical Center in Palo Alto, California focused on the use of robots to assist people with severe physical limitations to independently manipulate their own personal space ${ }^{2}$. Since then many prototype rehabilitation robots including occupational therapy robots have been built, tested, and evaluated. Most noteworthy rehabilitation robotic systems developed include the John Hopkins Robotic Arm/Work Table ${ }^{3)}$, the VA/ Stanford Robotic Workstation mentioned above, and the Vanderbilt ISAC system ${ }^{4}$. Because of high costs and limited capabilities, most rehabilitation robots are not yet commercially available.

\section{平成5年1月11日受付}

* Center for Intelligent Systems

Vanderbilt University

Nashville, Tennessee 37235, U.S.A.

Key Words : Rehabilitation Robot, United States, History,

Trends, R\&D Issues

\section{EVOLUTION OF ROBOTS FOR REHA- BILITATION}

This section briefly reviews the evolution of rehabilitative robots, noting how the development of robot technology has been mirrored in this specific branch of application. The major steps in the development of robotics has been teleoperation, motion planning, sensory fusion, reactive or reflex control, and learning.

Various rehabilitation robots with increasing sophistication in capabilities have been developed. In the United States the general purpose robotic arm is considered to be better than a collection of special purpose devices, each designed to perform a small subset of manipulative tasks.

\subsection{Teleoperated Manipulators}

Teleoperated manipulators are those whose joint motions are in direct, real-time control of the user. Typically, the user moves a mechanical "input device" with the necessary degrees of freedom. The motion sensed by the transducers of the input device are sent to the manipulator, which duplicates these joint motions. The main modification necessary for teleoperation by the handicapped is in the design of the input device.

The arm designed by JPL and John Hopkins University for high spinal cord injured persons uses chin motion input because of its good resolution ${ }^{5)}$. This could be used to control both the wheelchair (when away from the arm workstation) and the arm (when at the workstation). A sip and puff controller was also 
available for those who cannot provide input to the chin controller.

The arm motions performed through teleoperation could also be recorded for later playback in most of these robots. This had proved to be a useful feature for industrial robots. However, in the environment where a rehabilitation robot operates, repetitive motions are less likely. This calls for robots which can be instructed with a higher level-language where the path to the destination is generated by the robot.

\subsection{Manipulators with Programmed Motion}

Motion programming is an off-line mechanism for specifying the manipulator's task. Unlike teleoperation where the entire path has to be specified, only the initial and final arm position and orientation need to be specified. The intermediate points can then be generated by the motion planning system.

The Stanford Robotic Aid project which started in the early 80 s has developed many generations of rehabilitative robotic systems using industrial manipulators $^{2,6)}$. It uses the task programming language VAL to control the arm movements. The user can command the system to perform one of the preprogrammed tasks by voice. The location of objects to be manipulated, such as microwave oven, refrigerator, spoon, cup, and so forth, are either in predefined places known to the task program or given by the user by moving the arm. Extensive clinical evaluations have also been report$\mathrm{ed}^{7}$. Figure 1 shows the components of a generic Stanford-type robotic system.

\subsection{Manipulators with Sensors}

Rehabilitative robots are expected to operate in the unstructured world. The locations of the various objects cannot be guaranteed with the preciseness common in an industrial workcell. In addition, the presence of other humans means that the location of an

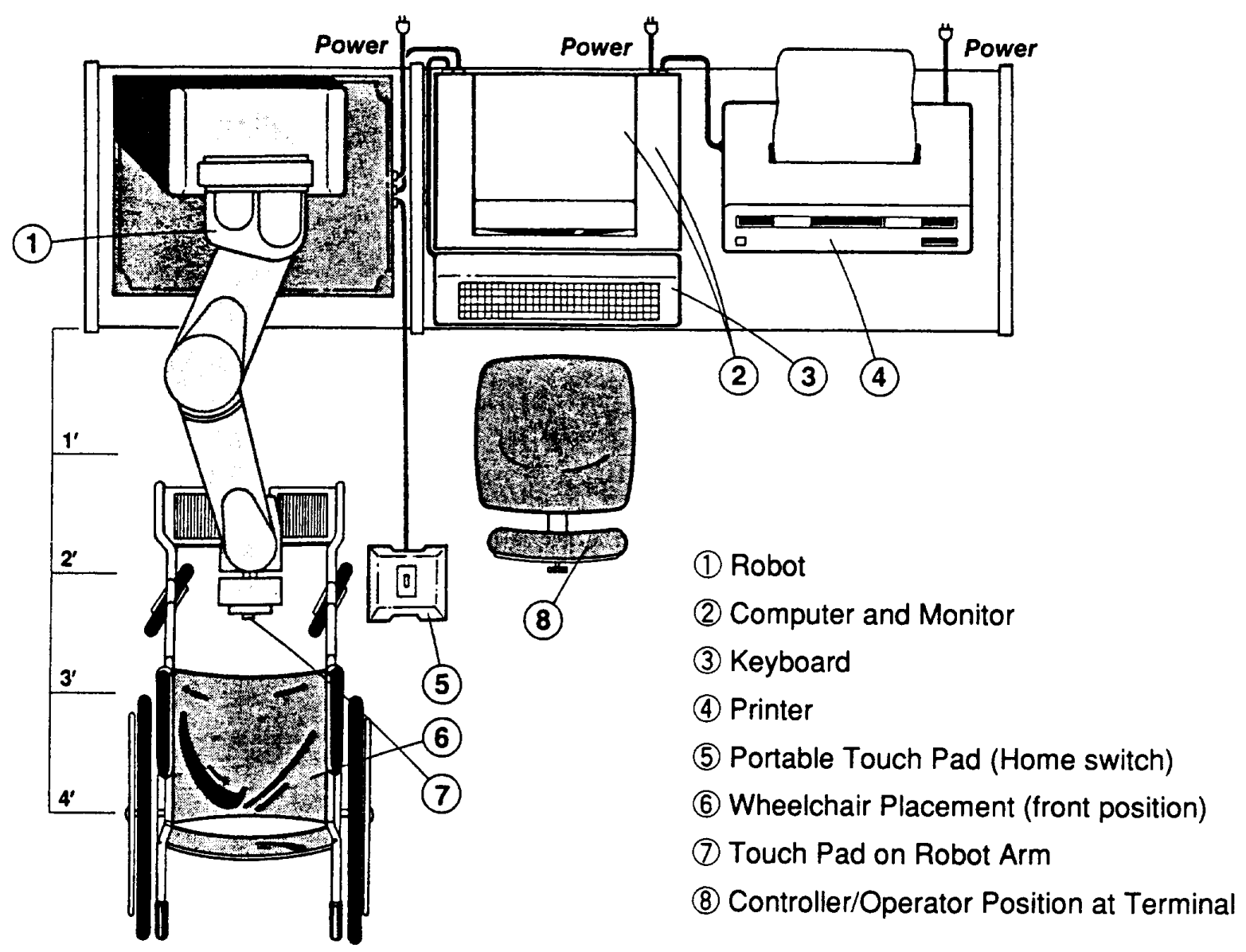

Fig. 1 


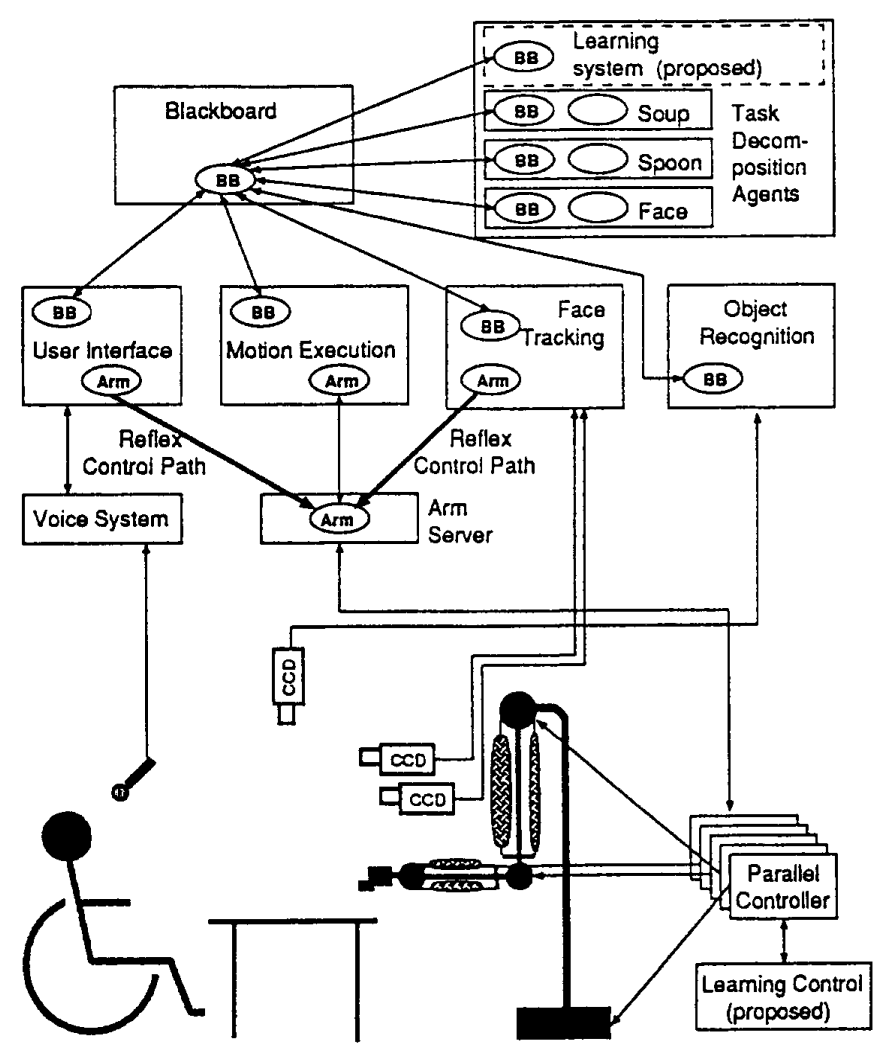

Fig. 2

object may change without the robot being aware of it. Therefore it is necessary for the robotic system to monitor the environment continuously. The most common sensor used for such monitoring activities is vision. Other sensors such as optical sensors, ultrasonic sensors and force sensors are also valuable.

The Stanford Robotic Aid project mentioned earlier reported research in progress on the use of various sensors including an ultrasonic obstacle avoidance system and a scanning laser ranging system. The Intelligent Soft Arm Control (ISAC) system being developed at Vanderbilt University uses a multi-camera vision system to recognize objects and real-time face tracking ${ }^{8}$. ISAC is an intelligent robotic aid for feeding the physically handicapped. The feeding task requires the recognition and location of objects such as spoon, fork, and bowl on the table so that the robot arm can manipulate them. These objects are recognized from an image taken by an overhead camera. ISAC also uses stereo cameras to track the movement of the user in real time. Figure 2 illustrates the integrated hardware/software configuration of the ISAC system.

\section{KEY R\&D ISSUES FOR ROBOTICS IN REHABILITATION}

Figure 3 shows the essential and desirable features needed for various tasks that a rehabilitative robot could perform. These features identify the key research and development issues in rehabilitation robots. Some of these issues have been around for a long time and a sufficient degree of success has been achieved. Examples are teleoperation and motion planning. Others are still under active research.

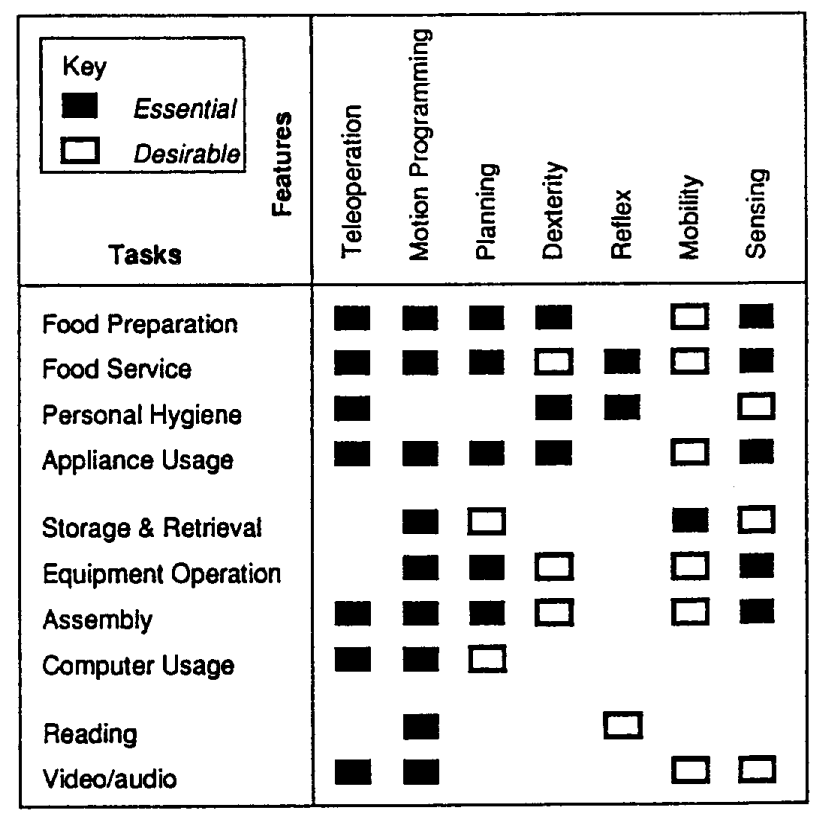

Fig. 3

\subsection{Planning}

Planning is a process of selecting actions that achieve the desired goals. It is a familiar human cognitive activity. This activity has been studied by AI researchers since the 1960s. STRIPS developed by Stanford Research Institute was one of the first and most influential planning systems. An excellent review of STRIPS and other planning systems is presented by Tale et $\mathrm{al}^{9}{ }^{9}$.

Planning will become necessary as more complex 
rehabilitative tasks are attempted. An example of such a complex task is food preparation and eating. It involves the combination of simpler actions such as using a spoon, a fork, or a knife, operating a microwave oven, and pouring liquids. Often, the same task can be achieved by various action sequences. The presence of the user makes the planning environment different from those where robots are expected to be more autonomous. The planner must react not only to unexpected changes in the environment, but also to additional user input. Vanderbilt ISAC system employs a distributed object-oriented planning system to accommodate such needs ${ }^{10}$.

\subsection{Dexterous Manipulators}

Most commercial manipulators have two fingered or parallel jaw grippers. This will pose severe limitations while performing rehabilitative tasks which require the dexterity of a human hand. Tasks ranging from using a fork to operating appliances such as microwave ovens, require more dexterity than that offered by simple grippers.

The Utah-MIT hand is one of the best-known dexterous mechanical hands ${ }^{11}$. Similar three-fingered robot hands were developed by teams at Stanford-JPL and others. Recently the Center for Advanced Technology in Automation and Robotics at RPI in Troy, New York developed an anatomically correct, fully functional, robot hand with five fingers ${ }^{12}$.

\subsection{Mobility}

Adding mobility to the robotic system extends the working volume and functions of the whole system beyond the desk-top work station environment. Several groups in the United States and Canada are working on adding this facility to their systems. One example is a mobile robotic aid system being developed at Rice University. A HIRO 2000 robot has been modified such that its workspace extends from the floor to table top heights and is controlled via a wireless communication link ${ }^{14)}$.

Another approach for robot mobility is based on mounting the robot manipulator arm on the patient's wheelchair. In such systems, the motion of the arm is completely controlled by the patient on the wheelchair. This combination offers a functional compensation for the impairment of both mobility and upper limb functions. An example of such systems is the MANUS wheelchair-borne manipulator ${ }^{14)}$ being developed in Canada. MANUS software provides the facility of special tasks preprogramming in addition to a library of standard control configurations with adaptive parameters that can be configured for different users with different disabilities.

\subsection{Sensing}

For any robotic aid system, sensors will be needed to guide the robot to achieve various tasks. It may well be sensory perception that will determine a success or a failure of a rehabilitation robot. If we humans were forced to select one sensor and exclude all others, it would surely be vision. Although machine or computer vision does not come close to matching human vision, robots can still benefit immensely from vision. Machine vision is one of the most actively studied areas in robotics and it is beyond the scope of this article to cover this field. Instead we will discuss one emerging area of machine vision called "active vision".

The use of vision in a robotic system was introduced in the mid 70s. Since then, the so-called passive vision techniques were used. One major problem with the traditional approach was that the computational cost was too high. This was an obstacle toward achieving a robust, real-time vision system. Recently, a growing number of researchers have started to adopt a new approach called active vision ${ }^{15,16,17)}$.

The name "active vision" was chosen to describe the ability of actively controlling the camera parameters, and more generally to combine vision with behavior to achieve robust, real-time perception for a robot interacting with a complex, dynamic world. In this paradigm, vision is most readily understood in the context of the visual behaviors that the system is engaged in. These behaviors may not require elaborate categorical representations of the 3-D world. 
One main use of active vision is in navigation and servoing. Those two operations are often needed by severely handicapped persons using an automated wheelchair. The wheelchair can be equipped with a camera used for navigation. One possible scenario of the use of such a system is the following: Through a gaze tracking device, the user gives a command to the system to move to a specific room. This action is then performed by the wheelchair by visually avoiding obstacles along the way. The camera will be moving and will need to actively adjust its focus and to maintain a view of the pathway of interest.

One sub-area of active vision is called foveation action. Human retina is known to produce an image with high resolution around the optical center (the fovea) and progressively lower resolution radially outwards from the fovea. This serves in significantly reducing the information content of the image to be processed. An algorithm simulating the foveation action is being implemented for the ISAC system $^{18)}$.

A more detailed discussion of important research issues in active vision are found in the paper by Swain and Sticker ${ }^{19}$.

\subsection{Reflex Control}

Reflex actions in biological systems are defined as actions which are triggered by a specific sensory input pattern and that run to completion without further intervention from higher centers. These reflex actions can be divided into the following categories: synthesis reflex actions such as locomotion, and protective re-

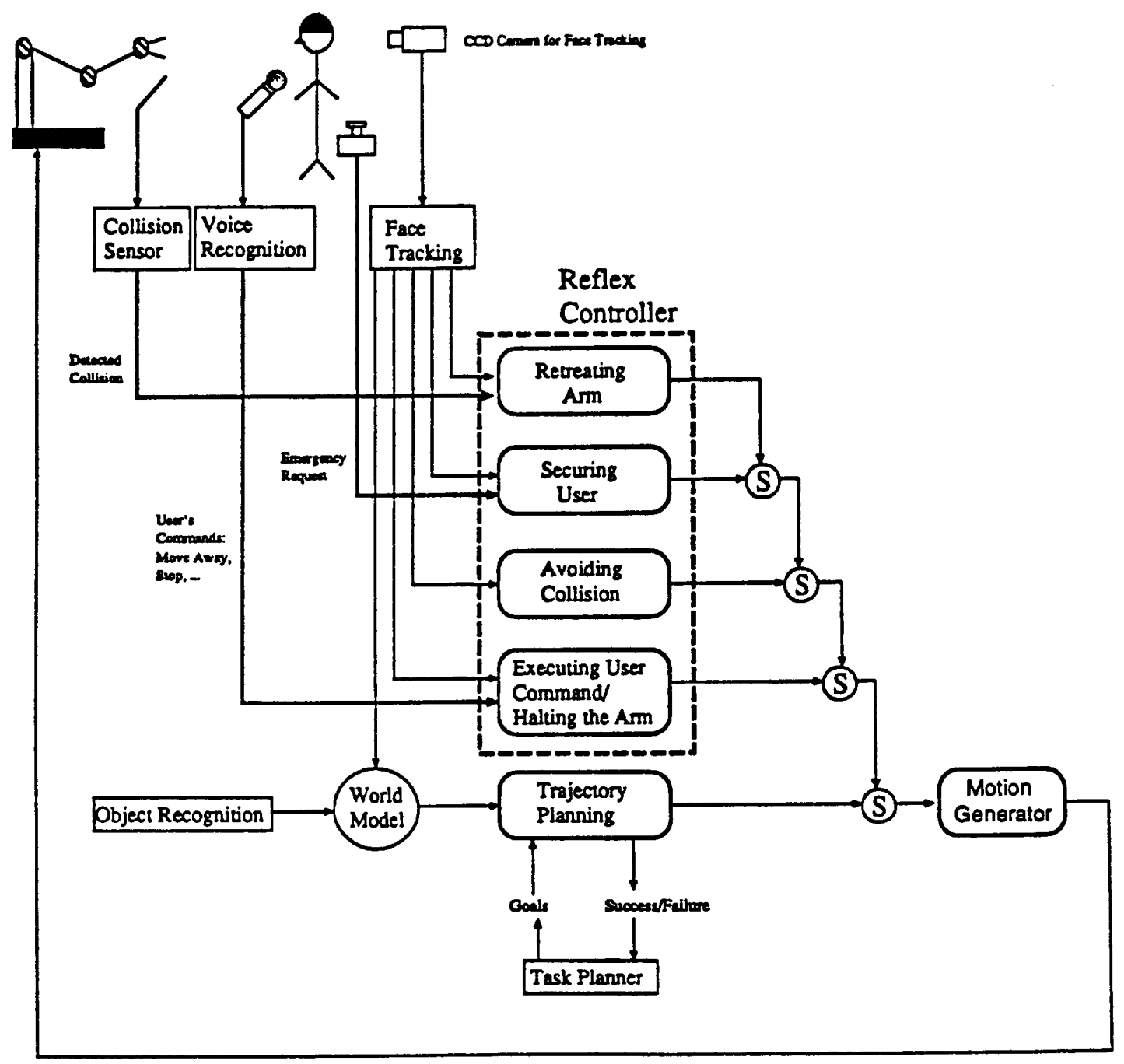

(S) = Suppressor

Fig. 4 
flex actions such as quick reactions to a potentially harmful situation.

Synthesis reflex actions were used by Bekey and Tomovic $^{20)}$ as a robot control tool for an artificial human-like leg used in rehabilitative robotics. The entire functional motion of the mechanism is modeled by a finite state machine with joint state transitions triggered by sensory information.

In the case of protective reflex actions, a reflex controller is used. It analyzes information from sensors and quickly decides on what kind of reflex action should be taken depending on the situation. Due to strict time constraints, most protective reflex actions bypass the planner in the robotic system. The controller then takes precedence over the actions of the highlevel modules. Protective reflex controllers have been implemented in the Vanderbilt ISAC system ${ }^{21)}$. This is shown in Figure 4.

\section{FUTURE TRENDS}

Ultimately the success of a robotic system for rehabilitation depends on its acceptance by users ${ }^{22)}$. Two major areas that will determine this acceptance are user interface and intelligence. Important research issues within these respective areas include natural language understanding and learning. This section discusses these issues in the context of rehabilitative applications.

\subsection{User Interface}

Voice is the most natural form of communication, especially suitable for a user with disabled arms. Currently, many commercially available speech recognition and synthesis systems exist. These have to be trained with a set of spoken phrases and their corresponding commands. The speech recognition algorithm is speaker dependent and is very sensitive to slight inflections of voice and to background noise.

These deficiencies create the need for a more sophisticated speech recognition system. SRI International has developed DECIPHER, a robust speech recognition system ${ }^{23)}$. It is unaffected by variations in voice characteristics, microphone characteristics, and background noise. It also has the ability to process continuous speech rather than isolated words. The recognition is based on a hidden Markov model using a language model based on a state-transition network. Other speaker independent speech recognition systems are being designed by research institutes such as MIT, Carnegie Mellon, AT\&T, and $\mathrm{BBN}^{24)}$ and some are commercially available.

For those who cannot use voice, there are a number of computer-aided systems which can be operated by the movement of eyes. Simply by looking at control keys displayed on a computer monitor screen, the user can perform a variety of simple functions including environmental control (controlling lights, televisions, etc.), playing games and running software ${ }^{25)}$.

\subsection{Learning and Adaptation}

While a good user interface is necessary for the acceptance of robots in rehabilitation, it will only be effective if the underlying system has a certain degree of intelligence. An important manifestation of intelligence is the ability to learn and adapt. One learning mechanism suitable for rehabilitation tasks is explanation-based learning $(\mathrm{EBL})^{26}$. When a given goal is achieved by a sequence of actions, the system tries to generalize this into a rule that can be applied to other situations. Consider the goal of feeding soup to the user. As a result of planning, the system generates an action sequence of gripping a spoon and dipping it into the bowl to get some soup. This plan can then be generalized by EBL into a general rule about using a spoon to get any liquid from a bowl. To do this, some knowledge of the domain must be known a priori.

Another form of learning is being developed for the Vanderbilt ISAC system. This mechanism requires that it should be made aware of all the conditions in the environment. Examples of such conditions are spoon in gripper, soup on spoon, available soup, etc. In addition, the robot should possess some basic preprogrammed actions such as being able to pick up a spoon, dip the spoon into the bowl, and moving the 
arm towards the mouth. In such a setup, the learning system can detect relations between actions and the conditions. Every action has a set of pre-conditions that must be true for successful operation. Also, every action results in the change of a set of conditions: the effects. The task of the learning system is to discover these preconditions and effects for all its actions. For tasks that are new to the system, the user instructs the robot to perform a sequence of actions. So the initial planning is done externally, by the user. As the robot performs these actions, it monitors the condition changes and forms the preconditions and effects relations with the actions. In this manner, knowledge about the task domain can be gathered. This can then be used for other related tasks ${ }^{27}$. A block diagram of the proposed system is shown in Figure 5.

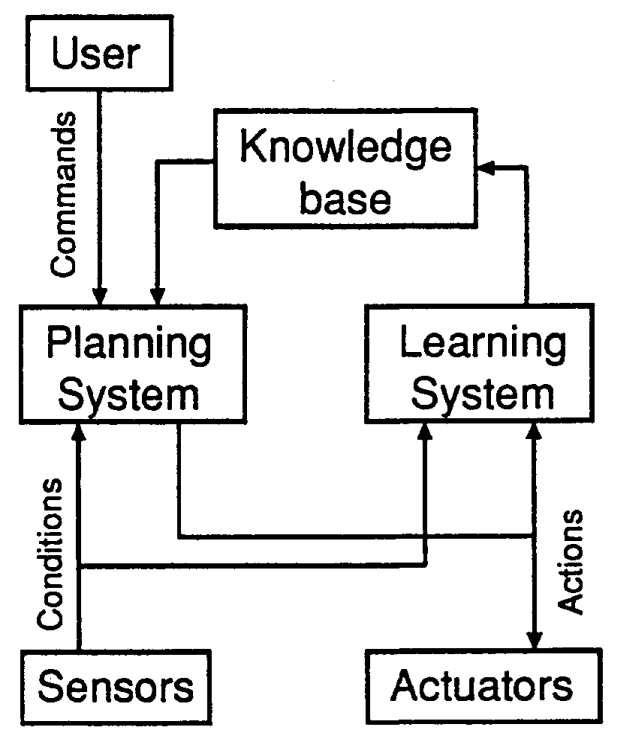

Fig. 5

\section{CONCLUSIONS}

Trends in rehabilitation robots in the United States were reviewed and key research and development issues were described using existing systems. Many research and development issues in rehabilitation robots are difficult to solve but a great deal of technical progress is expected in the next five years. The biggest obstacle to a wide usage of robotic systems in rehabil- itation will be costs.

\section{REFERENCES}

1) McNeal, D. R. : Rehabilitation engineering : Applying technology to help the disabled. IEEE Engineering in Medicine and Biology. $12: 15-16$ (1982)

2) LeBlanc, M. and Leifer, L. : Environmental control and robotic manipulation aids. IEEE Engineering in Medicine and Biology. 12 : 16-19 (1982)

3) Schmeisser, G. and Seamone, G. : A computeraided robotic arm/worktable system for the highlevel quadriplegic. $1: 41-47$ (1981)

4) Kawamura, K. and Fashoro, M. : Biorobotics. Technical Report, Center for Intelligent Systems, Vanderbilt University. CIS-92-05 (1992)

5) Seamone, W. and Schmeisser, G. : Evaluation of the APL/JHU robot arm workstation, in Interactive Robotic Aids - One Option for Independent Living : An International Perspective (R. Foulds, ed.). World Rehabilitation Fund, New York. 2933 (1986)

6) Van der Loos, H. F. M. et al. : Design of an omnidirectional mobile robot as a manipulation aid for the severely disabled, in Interactive Robotic Aids - One Option for Independent Living : An International Perspective (R. Fouls, ed.). World Rehabilitation Fund, New York. 61-63 (1986)

7) Hammel, J. et al. : Clinical evaluation of a desktop assistant. J. of Rehabilitation Research and Development. 26(3) : 1-16 (1989)

8) Kara, A. et al. : Reflex Control of a Robotic Aid System for the Physically Disabled. IEEE Control Systems Magazine. 12 : 71-77 (1992)

9) Tate, A., Hendler, J., and Drummond, M. : A review of AI planning techniques, in Readings in Planning. Morgan Kaufmann, San Mateo, CA. 26-49 (1990)

10) Bagchi, S. and Kawamura, K. : An architecture of a distributed object-oriented robotic system. 
IEEE/RSJ International Conference on Intelligent Robotics and Systems (IROS 92) (1992)

11) Russell, R. A. : Robot Tactile Sensing. Prentice Hall, New York. 122-124 (1990)

12) Society for Manufacturing Engineers: Robotics Today. (1992)

13) Regalbuto, M. A. et al. : Toward a practical mobile robotic aid system for people with severe physical disabilities. J. of Rehabilitation Research and Development. 29(1) : 19-26 (1992)

14) Kwee, H.H. et al. : The MANUS wheelchairborne manipulator. First International Workshop on Robotic Applications in Medical and Health Care, Ottawa. (1988)

15) Bajcsy, R. : Active perception. Proceedings of the IEEE. 76 : 996-1005 (1988)

16) Aloimonos, J. et al. : Active vision. International J. of Computer Vision. 1 : 333-356 (1988)

17) Ballard, D. : Animate vision. Artificial Intelligence. 48 : 57-86 (1991)

18) Bishay, M. : Controlled foveation for 3-D polyhedral object recognition. Master's thesis, department of Electrical Engineering, Vanderbilt University. (1992)

19) Swain, M.J. and Sticker, M. : Promising directions in active vision. Technical Report, University of Chicago. (1991)

20) Bekey, G. A. and Tomovic, R. : Robot control by reflex actions. Proceedings of the IEEE International Conference on Robotics and Automation, 240-246 (1986)

21) El-Gamal, M. et al. : Reflex control for an intelligent Robots and Systems (IROS 92), 1347-1354 (1992)
22) Dijkers, M. P. et al. : Patient and staff acceptance of robotic technology in occupational therapy : A pilot study. J. of Rehabilitation and Development 28(2) : 33-34 (1991)

23) Cohen, M. et al. : The DECIPHER Speech Recognition System. Proceedings of IEEE ICASSP 1 : 125-176 (1986)

24) Price, P. et al. : Spoken Language System Integration and Development. Proceedings of the ICSLP (1990)

25) LC Technologies, Inc. : The Eyegaze Computer System. Fairfax, VA. (1992)

26) Dejong, G. and Mooney, R. : Explanation-based learning : An alternative view. Machine Learning $1: 145-176$ (1986)

27) Bagchi, S., Biswas, G., and Kawamura, K. : A network architecture for planning in uncertain domains. paper to be presented at the AAAI Annual Meeting, Washington, D. C. (1993)

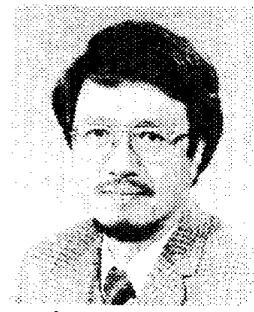

\section{河村和彦（かわむら かずひこ） \\ バンダービルト大学教授・日米先 端技術研究交流センター所長, 早 稲田大学工学部電気工学科理工学 部電気工学科（昭和 38 年）卒,} 41年ミシガン大学工学部講師, 48年からバッテ ル記念研究所研究員 